\title{
POLYNOMIAL INVERSE INTEGRATING FACTORS, FIRST INTEGRAL AND NON- EXISTENCE OF LIMIT CYCLES IN THE PLANE FOR QUADRATIC SYSTEMS
}

\author{
Ahmed M. Hussien
}

Dept. of Mathematics, College of Science, University of Duhok, Duhok, Kurdistan Region, Iraq

\section{ABSTRACT:}

The main purpose of this paper is to study the existence of polynomial inverse integrating factor and first integral, and non-existence of limit cycles for all systems. Furthermore, we consider some applications.

KEYWORDS: Polynomial inverse integrating factors, first integral, limit cycles.

\section{INTRODUCTION}

First we give some basic concepts and results about qualitative behaviour for autonomous ordinary differential equations

$$
\begin{aligned}
& \dot{x}=P(x, y), \\
& \dot{y}=Q(x, y),
\end{aligned}
$$

where $P(x, y)$ and $Q(x, y)$ are functions of class $C^{1}(\mathrm{U})$ and $\mathrm{U} \subseteq \mathbb{R}^{2}$. The periodic solution $\gamma$ of system (1) is an algebraic limit cycle if it is a limit cycle and contained in some irreducible invariant algebraic curve $\gamma=0$ of system (1), otherwise it's called non-algebraic limit cycle, (Calanchi, M. and Ruf, B., 2002) and (Chavarriga, J., Giacomini, H. and Gine , J., 1997).

Definition 1.1: A non-zero function V: $U \rightarrow R$ is said to be an inverse integrating factor of system (1)

in an open subset $\mathrm{U} \subseteq \mathbb{R}^{2}$ if $V \in \mathrm{C}^{1}(\mathrm{U}), V \neq 0$ in $\mathrm{U}$ that satisfies the equation

$$
P \frac{\partial V}{\partial x}+Q \frac{\partial V}{\partial y}=\left(\frac{\partial P}{\partial x}+\frac{\partial Q}{\partial y}\right) V
$$

In short notation, an inverses integrating factor of $\mathrm{V}(\mathrm{x}, \mathrm{y})$ system (1) satisfies

$$
\chi \mathrm{V}=\mathrm{V} \operatorname{div} \chi
$$

The inverse integrating factor is the most important tool in this work. We now give necessary conditions for a polynomial vector field to have a polynomial inverse integrating factor.

Definition 1.2: The system (1) is integrable on an open subset $U$ of $\mathbb{R}^{2}$ if there exists a non-constant analytic function $H: U \rightarrow \mathbb{R}$, called a first integral of the system on $U$, which is constant on all solutions curves $\phi(\mathrm{t})$ of system (1) contained in $U$; i.e., $H(\phi(\mathrm{t}))=$ constant for all values of $t$ for which the solution $\phi(\mathrm{t})$ is defined and contained in $U$.

Theorem 1.3 (Chavarriga, J., Giacomini, H. and Gine , J., 2000): The necessary and sufficient condition that a function $\mathrm{H}$ is a first integral of the system (1) on $\mathrm{U}$ is that

$$
\chi \mathrm{H}=\mathrm{P} \frac{\partial \mathrm{H}}{\partial \mathrm{x}}+\mathrm{Q} \frac{\partial \mathrm{H}}{\partial \mathrm{y}}=0 .
$$

One of the classical tools in the classification of all trajectories of a dynamical system is to find first integral. For two dimensional vector fields a first integral completely determines its phase portrait. But usually it is very difficult to detect a planar vector field is integrable or not.

We say that system (1) is integrable if $\operatorname{div}(\chi)=\frac{\partial P}{\partial x}+\frac{\partial Q}{\partial y}=0$. If system (1) is integrable, then there exists a function $\mathrm{H}$ such that $\frac{\partial \mathrm{H}}{\partial \mathrm{x}}=-\mathrm{Q}, \frac{\partial \mathrm{H}}{\partial \mathrm{y}}=\mathrm{P}$, then any such $\mathrm{H}$ is a first integral of system (1) (Ferragut, A., 2006).

Theorem 1.4 (Chavarriga, 2004.): Let $\mathrm{H}: \mathrm{U} \rightarrow \mathbb{R}$ be the first integral of a system (1). Then system (1) has no limit cycle contained in $\mathrm{U}$.

The following theorem gives an important relation between inverse integrating factor and first integral.

Theorem 1.5 (Ferragut, A., 2006): Let $V$ be an inverse integrating factor of system (1) defined in the open subset $U$ 드 $\mathbb{R}^{2}$. Then,

1) The function $\frac{1}{V}$, defined in $U \backslash\{V=0\}$ is an integrating factor of system (1). Moreover; the function

$H(x, y)=-\int \frac{P(x, y)}{V(x, y)} d y+\int\left(\frac{Q(x, y)}{V(x, y)}+\frac{\partial}{\partial x} \int \frac{P(x, y)}{V(x, y)} d y\right) d x$ is a first integral of (1).

2) If system (1) has a first integral $H$, then the function $V_{H}(x, y)=\frac{p}{-\frac{\partial H}{\partial y}}=\frac{Q}{\frac{\partial H}{\partial x}} \quad$ is an inverse integrating factor of (1). Moreover, the system $\dot{x}=\frac{p}{V_{H}}=-\frac{\partial H}{\partial y}, \dot{y}=\frac{Q}{V_{H}}=\frac{\partial H}{\partial x} \quad$ is Hamiltonian in $U \backslash\{V=0\}$.

Theorem 1.6 (Ferragut, A., 2006): Let V: $U \rightarrow \mathbb{R}$ be an inverse integrating factor of (1). If $\gamma \subset \mathrm{U}$ is a limit cycle of (1), then $\gamma$ is contained in the set

$$
\Sigma=\{(\mathrm{x}, \mathrm{y}) \in \mathrm{U}: \mathrm{V}(\mathrm{x}, \mathrm{y})=0\} .
$$

The existence of limit cycles was first detected by Poincare.

Definition 1.7: A closed trajectory $\phi$ in a phase portrait is called a limit cycle if it is isolated from all other closed trajectories more precisely, if there is a neighborhood of $\phi$ which contains no other closed trajectories.

Definition 1.8: The geometrical representation of the qualitative behaviour of (1) is called the phase-portrait.

Theorem 1.9 (Chavarriga, J., Llibre , J. and Sorolla , J., 2001): If the system (1) has no singular point, then it has no limit cycles. 
Definition 1.10: A singular point for system (1) is a point $\left(\mathrm{x}_{0}, \mathrm{y}_{0}\right)$ such that $\mathrm{f}\left(\mathrm{x}_{0}, \mathrm{y}_{0}\right)=\mathrm{g}\left(\mathrm{x}_{0}, \mathrm{y}_{0}\right)=0$, a singular point is a particular case of solution, where $\phi_{\mathrm{t}}\left(\mathrm{x}_{0}, \mathrm{y}_{0}\right)=\left(\mathrm{x}_{0}, \mathrm{y}_{0}\right)$ for all $t \in \mathbb{R}$.

Definition 1.11: The associated linearized system (4) at singular point $\left(\mathrm{x}_{0}, \mathrm{y}_{0}\right)$ is given by the Jacobian matrix $J_{\left(x_{0}, y_{0}\right)}$ where

and the system

$$
J_{\left(x_{0}, y_{0}\right)}=\left.\left(\begin{array}{ll}
\frac{\partial P}{\partial x} & \frac{\partial P}{\partial y} \\
\frac{\partial Q}{\partial x} & \frac{\partial Q}{\partial y}
\end{array}\right)\right|_{(x, y)=\left(x_{0}, y_{0}\right)}
$$

$$
\left(\begin{array}{l}
\dot{x} \\
\dot{y}
\end{array}\right)=J_{\left(x_{0}, y_{0}\right)}\left(\begin{array}{l}
x \\
y
\end{array}\right)
$$

is said to be the linearization (or linearized system).

\section{POLYNOMIAL INVERSE INTEGRATING FACTORS AND FIRST INTEGRALS}

In this section we give some results about the existence of polynomial an inverse integrating factors and First integrals for Quadratic polynomial systems.

In the first part of this work the Quadratic systems are the polynomial real differential systems of type (1) of degree $m=2$. Our main objective is to classify some Quadratic systems having a polynomial inverse integrating factor $V(x, y)$.

Our result in this section is the following.

2.1 Method for computing polynomial inverse integrating factors for Quadratic system

Consider the real planar Quadratic system

$$
\begin{gathered}
\dot{x}=\sum_{j=0}^{2} \sum_{i=0}^{j} a_{i, j-i} x^{i} y^{j-i}=P_{2}(x, y) \\
\dot{y}=\sum_{j=0}^{2} \sum_{i=0}^{j} b_{i, j-i} x^{i} y^{j-i}=Q_{2}(x, y)
\end{gathered}
$$

we assume that $P_{2}$ and $Q_{2}$ have no common factors; otherwise the system can be transformed into a quadratic one. In order to find a polynomial inverse integrating factor of this system. We denoted this equationby (2).

Next we describe the method we use to find polynomial inverse integrating factors of degree $k>0$.

Method 1. Since we are looking for real polynomial inverse integrating factors of degree $k \in \mathbb{N}$, we write $V(x, y)$ as

$$
V(x, y)=\sum_{i+j=0}^{k} V_{i, j} x^{i} y^{j},
$$

where $V_{i, j} \in \mathbb{R}$. Equation (2) is a polynomial equation , since $P_{2}(x, y), Q_{2}(x, y)$ and $V$ are polynomial functions, and it can be written as a linear system with unknowns $V_{i, j}, i+j=$ $0, \ldots, k$.

\subsection{Existence of inverse integrating factor of Quadratic} polynomial systems:

Theorem 2.2: The linear system

$$
\begin{aligned}
& \dot{x}=a_{1} x+b_{1} y \\
& \dot{y}=a_{2} x+b_{2} y
\end{aligned}
$$

has inverse integrating factor $V(x, y)=a_{2} x^{2}+\left(b_{2}-\right.$ $\left.a_{1}\right) x y-b_{1} y^{2}$ and if $a_{1} \neq b_{2}$ the first integral is

$$
\begin{aligned}
& H(x, y) \\
& =\frac{1}{2} \ln \left(-a_{2} x^{2}-x y b_{2}+x y a_{1}+b_{1} y^{2}\right) \\
& \left.+\frac{\arctan \left(\frac{-x b_{2}+a_{1} x+2 b_{1} y}{\sqrt{-4 a_{2} x^{2} b_{1}-x^{2} b_{2}^{2}+2 x^{2} b_{2} a_{1}-a_{1}^{2} x^{2}}}\right) a_{1} x}{\sqrt{-4 a_{2} x^{2} b_{1}-x^{2} b_{2}^{2}+2 x^{2} b_{2} a_{1}-a_{1}^{2} x^{2}}}\right) x b_{2} \\
& +\frac{\arctan \left(\frac{-x b_{2}+a_{1} x+2 b_{1} y}{\sqrt{-4 a_{2} x^{2} b_{1}-x^{2} b_{2}^{2}+2 x^{2} b_{2} a_{1}-a_{1}^{2} x^{2}}}\right)}{\sqrt{-4 a_{2} x^{2} b_{1}-x^{2} b_{2}^{2}+2 x^{2} b_{2} a_{1}-a_{1}^{2} x^{2}}}
\end{aligned}
$$

Proof: let $V(x, y)$ be an inverse integrating factor then by Definition1.1from eq. (2) is

$$
\begin{gathered}
P \frac{\partial V}{\partial x}+Q \frac{\partial V}{\partial y}=\left(\frac{\partial P}{\partial x}+\frac{\partial Q}{\partial y}\right) V \\
\left(a_{1} x+b_{1} y\right) \frac{\partial V}{\partial x}+\left(a_{2} x+b_{2} y\right) \frac{\partial V}{\partial y}=\left(a_{1}+b_{2}\right) V
\end{gathered}
$$

Solving quasi linear equation(6) we have

From (1) and (2)

$$
\frac{d x}{a_{1} x+b_{1} y}=\frac{d y}{a_{2} x+b_{2} y}=\frac{d V}{\left(a_{1}+b_{2}\right) V}
$$

$$
\begin{gathered}
\frac{d x}{a_{1} x+b_{1} y}=\frac{d y}{a_{2} x+b_{2} y} \\
\left(a_{1} x+b_{1} y\right) d y-\left(a_{2} x+b_{2} y\right) d x=0
\end{gathered}
$$

We get homogenous O.DE

$$
\frac{d y}{d x}=\frac{a_{2} x+b_{2} y}{a_{1} x+b_{1} y}
$$

by definition of homogenous, let $w=\frac{y}{x}, y=w x$

$$
\begin{gathered}
d y=w d x+x d w \text { or } \frac{d y}{d x}=w+x \frac{d w}{d x} \\
w+x \frac{d w}{d x}=\frac{a_{2} x+b_{2} w x}{a_{1} x+b_{1} w x} \\
w+x \frac{d w}{d x}=\frac{x\left(a_{2}+b_{2} w\right)}{x\left(a_{1}+b_{1} w\right)} \\
x \frac{d w}{d x}=\frac{a_{2}+b_{2} w}{a_{1}+b_{1} w}-w=\frac{a_{2}+b_{2} w-a_{1} w-b_{1} w^{2}}{a_{1}+b_{1} w} \\
\frac{d x}{x}=\frac{a_{1}+b_{1} w}{a_{2}+\left(b_{2}-a_{1}\right) w-b_{1} w^{2}} d w
\end{gathered}
$$

Eq. (3) is separable equation

To solve Eq. (3) it is difficult integral for

$$
\begin{aligned}
& \int \frac{a_{1}+b_{1} w}{a_{2}+\left(b_{2}-a_{1}\right) w-b_{1} w^{2}} d w \\
& =-\frac{1}{2} \frac{1}{\sqrt{-a_{2} b_{1}-b_{2}^{2}+2 b_{2} a_{1}-a_{1}^{2}}}\left(\left(2 a_{1}\right.\right. \\
& \left.+2 b_{2}\right) \arctan \left(\frac{-b_{2}+a_{1}+2 b_{1} w}{\sqrt{-a_{2} b_{1}-b_{2}^{2}+2 b_{2} a_{1}-a_{1}^{2}}}\right)+\ln \left(b_{1} w^{2}\right. \\
& \left.\left.+\left(-b_{2}+a_{1}\right) w-a_{2}\right) \sqrt{-a_{2} b_{1}-b_{2}^{2}+2 b_{2} a_{1}-a_{1}^{2}}\right)
\end{aligned}
$$

Eq. (3) has solution

$$
\begin{aligned}
& \ln (x) \\
& +\frac{1}{2} \frac{1}{\sqrt{-4 a_{2} b_{1}-b_{2}^{2}+2 b_{2} a_{1}-a_{1}^{2}}}\left(\left(2 a_{1}\right.\right. \\
& \left.+2 b_{2}\right) \arctan \left(\frac{-b_{2}+a_{1}+2 b_{1} w}{\sqrt{-4 a_{2} b_{1}-b_{2}^{2}+2 b_{2} a_{1}-a_{1}^{2}}}\right) \\
& +\ln \left(b_{1} w^{2}+\left(-b_{2}+a_{1}\right) w\right. \\
& \left.-a_{2}\right) \sqrt{-4 a_{2} b_{1}-b_{2}^{2}+2 b_{2} a_{1}-a_{1}^{2}}=\ln (c)
\end{aligned}
$$

substitute $w=\frac{y}{x}$ in solution, we get 


$$
\begin{aligned}
& \ln (x)+\ln (c) \\
& +\frac{1}{2} \frac{1}{\sqrt{-4 a_{2} b_{1}-b_{2}^{2}+2 b_{2} a_{1}-a_{1}^{2}}}\left(2 a_{1}\right. \\
& \left.+2 b_{2}\right) \arctan \left(\frac{-b_{2}+a_{1}+\frac{2 b_{1} y}{x}}{\sqrt{-4 a_{2} b_{1}-b_{2}^{2}+2 b_{2} a_{1}-a_{1}^{2}}}\right) \\
& +\ln \left(\frac{b_{1} y^{2}}{x^{2}}+\frac{\left(-b_{2}+a_{1}\right) y}{x}\right. \\
& \left.\left.-a_{2}\right) \sqrt{-4 a_{2} b_{1}-b_{2}^{2}+2 b_{2} a_{1}-a_{1}^{2}}\right)=0
\end{aligned}
$$

It is difficult to find $y$ in the above solution From Eq. (1) and Eq. (3), we have this

$$
\frac{d x}{a_{1} x+b_{1} y}=\frac{d V}{\left(a_{1}+b_{2}\right) V}
$$

substitute $y$ in eq.(4) and solve, we get $V(x, y)$, but also it is very difficult for solving. Hence, the method it is neglected Eq. (6) is in the form. By Method 1, for $k=2$, let $V(x, y)=$ $v_{20} x^{2}+v_{11} x y+v_{02} y^{2}$

$\left(a_{1} x+b_{1} y\right)\left(2 v_{20} x+v_{11} y\right)$

$$
\begin{aligned}
& +\left(a_{2} x+b_{2} y\right)\left(v_{11} x+2 v_{02} y\right) \\
& =\left(a_{1}+b_{2}\right)\left(v_{20} x^{2}+v_{11} x y+v_{02} y^{2}\right)
\end{aligned}
$$

$2 a_{1} v_{20} x^{2}+a_{1} v_{11} x y+2 b_{1} v_{20} x y+b_{1} v_{11} y^{2}+a_{2} v_{11} x^{2}$

$$
+2 a_{2} v_{02} x y+b_{2} v_{11} x y+2 b_{2} v_{02} y^{2}
$$$$
=\left(a_{1}+b_{2}\right) v_{20} x^{2}+\left(a_{1}+b_{2}\right) v_{11} x y
$$

$\left(2 a_{1} v_{20}+a_{2} v_{11}\right) x^{2}$

$$
+\left(a_{1}+b_{2}\right) v_{02} y^{2}
$$

$$
\begin{aligned}
& +\left(a_{1} v_{11}+2 b_{1} v_{20}+2 a_{2} v_{02}\right. \\
& \left.+b_{2} v_{11}\right) x y+\left(b_{1} v_{11}+2 b_{2} v_{02}\right) y^{2} \\
& =\left(a_{1}+b_{2}\right) v_{20} x^{2}+\left(a_{1}+b_{2}\right) v_{11} x y \\
& +\left(a_{1}+b_{2}\right) v_{02} y^{2}
\end{aligned}
$$

The coefficients of $x^{2}, x y$ and $y^{2}$ are:

$$
2 a_{1} v_{20}+a_{2} v_{11}=\left(a_{1}+b_{2}\right) v_{20}
$$$$
a_{1} v_{11}+2 b_{1} v_{20}+2 a_{2} v_{02}+b_{2} v_{11}=\left(a_{1}+b_{2}\right) v_{11}
$$

$$
b_{1} v_{11}+2 b_{2} v_{02}=\left(a_{1}+b_{2}\right) v_{02}
$$

From the above system, we get;

$$
\begin{gathered}
\left(a_{1}-b_{2}\right) v_{20}+a_{2} v_{11}+0 v_{02}=0 \\
b_{1} v_{20}+0 v_{11}+a_{2} v_{02}=0 \\
0 v_{20}+b_{1} v_{11}+\left(b_{2}-a_{1}\right) v_{02}=0 \\
\left(\begin{array}{ccc}
a_{1}-b_{2} & a_{2} & 0 \\
2 b_{1} & 0 & 2 a_{2} \\
0 & b_{1} & b_{2}-a_{1}
\end{array}\right)\left(\begin{array}{c}
v_{20} \\
v_{11} \\
v_{02}
\end{array}\right)=0 \\
A_{2}=\left(\begin{array}{ccc}
a_{1}-b_{2} & a_{2} & 0 \\
2 b_{1} & 0 & 2 a_{2} \\
0 & b_{1} & b_{2}-a_{1}
\end{array}\right) \\
A_{2} V^{2}=0,
\end{gathered}
$$

After solving the system

$$
\begin{aligned}
& b_{1} v_{11}=\left(a_{1}-b_{2}\right) v_{02} \text { and } v_{11}=\frac{a_{1}-b_{2}}{b_{1}} v_{02} \\
& \text { or } v_{02}=\frac{b_{1}}{a_{1}-b_{2}} v_{11} \\
& b_{1} v_{20}+\frac{a_{2} b_{1}}{a_{1}-b_{2}} v_{11}=0 \\
& v_{20}=\frac{-a_{2}}{a_{1}-b_{2}} v_{11}
\end{aligned}
$$

$v_{11}$ is independent constant

Substitute $v_{20}$ and $v_{02}$ in $V(x, y)$

$$
\begin{gathered}
V(x, y)=\left(\frac{-a_{2}}{a_{1}-b_{2}} v_{11} x^{2}+v_{11} x y+\frac{b_{1}}{a_{1}-b_{2}} v_{11} y^{2}\right) \\
V(x, y)=\frac{-v_{11}}{a_{1}-b_{2}}\left(a_{2} x^{2}+\left(b_{2}-a_{1}\right) x y-b_{1} y^{2}\right)
\end{gathered}
$$

Choose $v_{11}=-\left(a_{1}-b_{2}\right)$, we get the result

$$
V(x, y)=a_{2} x^{2}+\left(b_{2}-a_{1}\right) x y-b_{1} y^{2} \text {. }
$$

Theorem 2.3: The nonlinear system

$$
\begin{aligned}
& \dot{x}=a_{20} x^{2}+a_{11} x y+a_{02} y^{2} \\
& \dot{y}=b_{20} x^{2}+b_{11} x y+b_{02} y^{2}
\end{aligned}
$$

has inverse integrating factor $V(x, y)=b_{20} x^{3}+\left(b_{11}-\right.$ $\left.a_{20}\right) x^{2} y+\left(b_{02}-a_{11}\right) x y^{2}-a_{02} y^{3}$, if $a_{20} \neq b_{11}$.

Proof: let $V(x, y)$ be an inverse integrating factor then by Definition 1.1 from Eq. (2) is

$$
\begin{gathered}
P \frac{\partial V}{\partial x}+Q \frac{\partial V}{\partial y}=\left(\frac{\partial P}{\partial x}+\frac{\partial Q}{\partial y}\right) V \\
\left(a_{20} x^{2}+a_{11} x y+\right. \\
\left.+a_{02} y^{2}\right) \frac{\partial V}{\partial x}+\left(b_{20} x^{2}+b_{11} x y+b_{02} y^{2}\right) \frac{\partial V}{\partial y} \\
=\left(2 a_{20} x+a_{11} y+b_{11} x\right. \\
\left.+2 b_{02} y\right) V
\end{gathered}
$$

Solving quasi linear equation (8) we have

$$
\begin{gathered}
\frac{d x}{\left(a_{20} x^{2}+a_{11} x y+a_{02} y^{2}\right)}=\frac{d y}{\left(b_{20} x^{2}+b_{11} x y+b_{02} y^{2}\right)} \\
=\frac{d V}{\left(2 a_{20} x+a_{11} y+b_{11} x+2 b_{02} y\right) V}
\end{gathered}
$$

It is very difficult for solving this equation. Hence, the method it is neglected eq. (7) is in the form. By Method 1, for $k=3$, let $V(x, y)=v_{30} x^{3}+v_{21} x^{2} y+v_{12} x y^{2}+v_{03} y^{3}$.

$$
\begin{aligned}
\left(a_{20} x^{2}+a_{11} x y+\right. & \left.a_{02} y^{2}\right)\left(3 v_{30} x^{2}+2 v_{21} x y+v_{12} y^{2}\right) \\
& +\left(b_{20} x^{2}+b_{11} x y+b_{02} y^{2}\right)\left(v_{21} x^{2}\right. \\
& \left.+2 v_{12} x y+3 v_{03} y^{2}\right) \\
& =\left(2 a_{20} x+a_{11} y+b_{11} x+2 b_{02} y\right)\left(v_{30} x^{3}\right. \\
& \left.+v_{21} x^{2} y+v_{12} x y^{2}+v_{03} y^{3}\right)
\end{aligned}
$$

after calculations

The coefficients of $x^{4}, x^{2} y^{2}, x^{3} y, x y^{3}$ and $y^{4}$ are:

$$
\begin{gathered}
\left(a_{2,0}-b_{1,1}\right) v_{30}+b_{2,0} v_{21}=0 \\
\left(2 a_{1,1}-2 b_{0,2}\right) v_{30}+2 b_{2,0} v_{12}=0 \\
3 a_{0,2} v_{30}+\left(a_{1,1}-b_{0,2}\right) v_{21}+3 b_{2,0} v_{03}=0 \\
2 a_{0,2} v_{21}+\left(-2 a_{2,0}+2 b_{1,1}\right) v_{03}=0 \\
a_{0,2} v_{12}+\left(-a_{1,1}+b_{0,2}\right) v_{03}=0
\end{gathered}
$$

From the above system, we get;

$$
\begin{gathered}
\left(\begin{array}{cccc}
a_{2,0}-b_{1,1} & b_{2,0} & 0 & 0 \\
2 a_{1,1}-2 b_{0,2} & 0 & 2 b_{2,0} & 0 \\
3 a_{0,2} & a_{1,1}-b_{0,2} & 0 & 3 b_{2,0} \\
0 & 2 a_{0,2} & 0 & -2 a_{2,0}+2 b_{1,1} \\
0 & 0 & a_{0,2} & -a_{1,1}+b_{0,2}
\end{array}\right)_{5 \times 4}\left(\begin{array}{l}
v_{30} \\
v_{21} \\
v_{12} \\
v_{03}
\end{array}\right)=0 \\
A_{3}=\left(\begin{array}{cccc}
a_{2,0}-b_{1,1} & b_{2,0} & 0 & 0 \\
2 a_{1,1}-2 b_{0,2} & 0 & 2 b_{2,0} & 0 \\
3 a_{0,2} & a_{1,1}-b_{0,2} & 0 & 3 b_{2,0} \\
0 & 2 a_{0,2} & 0 & -2 a_{2,0}+2 b_{1,1} \\
0 & 0 & a_{0,2} & -a_{1,1}+b_{0,2}
\end{array}\right)_{5 \times 4} \\
A_{3} V^{3}=0,
\end{gathered}
$$

After solving the system

$$
\begin{gathered}
v_{30}=\frac{-b_{2,0}}{\left(a_{2,0}-b_{1,1}\right)} v_{21}, v_{12}=\frac{\left(a_{1,1}-b_{0,2}\right)}{\left(a_{2,0}-b_{1,1}\right)} v_{21}, \\
v_{03}=\frac{a_{0,2}}{\left(a_{2,0}-b_{1,1}\right)} v_{21}, v_{21}=-\left(a_{20}-b_{11}\right)
\end{gathered}
$$

Substitute $v_{30}, v_{12}, v_{03}$ and $v_{21}$ in $V(x, y)$, we get the result

$$
\begin{gathered}
V(x, y)=b_{20} x^{3}+\left(b_{11}-a_{20}\right) x^{2} y+\left(b_{02}-a_{11}\right) x y^{2} \\
-a_{02} y^{3}
\end{gathered}
$$




\section{APPLICATION IN A CHEMICAL REACTION OF NON-EXISTENCE OF LIMIT CYCLES FOR QUADRATIC SYSTEM}

\subsection{Two autocatalysing molecules that form a complex:}

we have two molecules $\mathrm{X}, \mathrm{Y}$ which induces their own production mediated by molecules $\mathrm{A}$ and $\mathrm{B} . \mathrm{X}$ and $\mathrm{Ycan}$ also form a complex $\mathrm{C}(=\mathrm{XY})$.Again assume that there is a surplus of $\mathrm{A}$ and $\mathrm{B}$, resulting in their Concentrations being constant. Since the dynamics of $\mathrm{X}$ and $\mathrm{Y}$ does not depend on the complex $\mathrm{C}$, it will also be left out of the analysis, illustrate of the system (9). A chemical reaction leading to a limit cycle: Consider the reaction

$$
\begin{aligned}
& X+Y \rightarrow 2 X \\
& A+X \rightarrow 2 X \\
& B+Y \rightarrow 2 Y
\end{aligned}
$$

This is a modification of a reaction system suggested by Schnakenberg (Mustafa, 2005).

Theorem 3.2: The relevant differential equations are

$$
\begin{gathered}
\dot{x}=x+a_{20} x^{2}+x y+a_{02} y^{2} \\
\dot{y}=-y-a_{02} x^{2}-x y-a_{20} y^{2}
\end{gathered}
$$

Where $a_{02}$ and $a_{20}$ are appropriate constant.

Proof: The fixed points of system (9) are

$$
\left(x_{0}, y_{0}\right)=(0,0),\left(\frac{-1}{a_{20}+1+a_{02}}, \frac{-1}{a_{20}+1+a_{02}}\right)
$$

The linearization of the system at $\left(x_{0}, y_{0}\right)$ is

$$
\begin{aligned}
& A=\left(\begin{array}{ll}
P_{x} & P_{y} \\
Q_{x} & Q_{y}
\end{array}\right)_{\left(x_{0}, y_{0}\right)} \\
& =\left(\begin{array}{cc}
1+2 a_{20} x+y & x+2 a_{02} y \\
-2 a_{02} x-y & -1-x-2 a_{20} y
\end{array}\right)_{\left(x_{0}, y_{0}\right)}
\end{aligned}
$$

So the matrix $A$ at $(0,0)$ become:-

$$
A=\left(\begin{array}{cc}
1 & 0 \\
0 & -1
\end{array}\right)
$$

and by characteristic equation we get: $\operatorname{det}(A-\lambda I)=0$

$$
\begin{gathered}
\left|\left(\begin{array}{cc}
1 & 0 \\
0 & -1
\end{array}\right)-\lambda\left(\begin{array}{ll}
1 & 0 \\
0 & 1
\end{array}\right)\right|=0 \\
\lambda= \pm 1
\end{gathered}
$$

the system (9) has a singular point at the origin and the linearization at the origin is

$$
\begin{aligned}
& \dot{x}=x, \\
& \dot{y}=-y
\end{aligned}
$$

and the phase portrait of the system (10) is

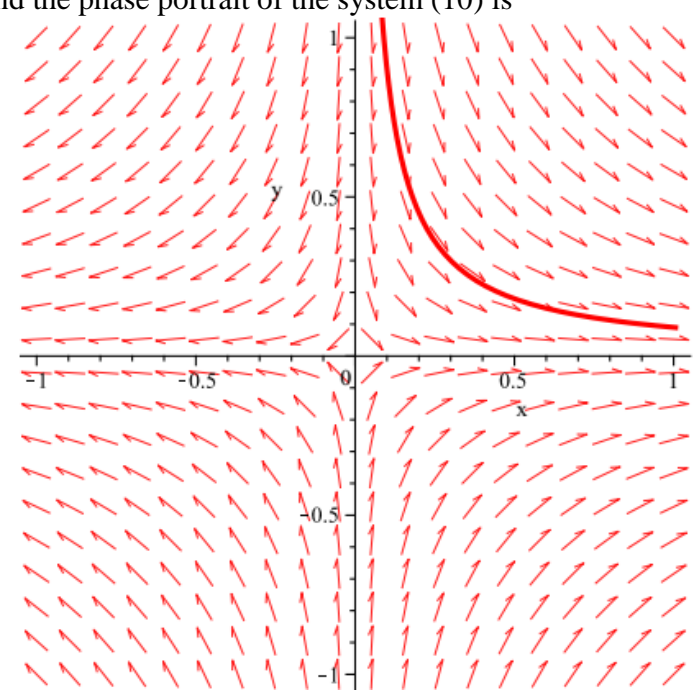

Figure 1. The phase portraits for the system (10)
So the matrix $A$ at $\left(\frac{-1}{a_{20}+a_{02}+1}, \frac{-1}{a_{20}+a_{02}+1}\right)$ become:

$$
A=\left(\begin{array}{cc}
\frac{a_{02}-a_{20}}{a_{20}+1+a_{02}} & \frac{-1-2 a_{02}}{a_{20}+1+a_{02}} \\
\frac{2 a_{02}+1}{a_{20}+1+a_{02}} & \frac{a_{20}-a_{02}}{a_{20}+a_{02}+1}
\end{array}\right)
$$

and by characteristic equation we get:

$$
\begin{aligned}
& \left|\left(\begin{array}{ll}
\frac{a_{02}-a_{20}}{a_{20}+1+a_{02}} & \frac{-1-2 a_{02}}{a_{20}+1+a_{02}} \\
\frac{2 a_{02}+1}{a_{20}+1+a_{02}} & \frac{a_{20}-a_{02}}{a_{20}+a_{02}+1}
\end{array}\right)-\lambda\left(\begin{array}{ll}
1 & 0 \\
0 & 1
\end{array}\right)\right|=0 \\
& \left(\frac{a_{02}-a_{20}}{a_{20}+1+a_{02}}-\lambda\right)\left(\frac{a_{20}-a_{02}}{a_{20}+1+a_{02}}-\lambda\right) \\
& -\left(\frac{-1-2 a_{02}}{a_{20}+1+a_{02}}\right)\left(\frac{2 a_{02}+1}{a_{20}+1+a_{02}}\right)=0 \\
& \frac{2 a_{02} a_{20}-\left(a_{02}\right)^{2}-\left(a_{20}\right)^{2}+\lambda^{2}\left(a_{20}+1+a_{02}\right)^{2}}{\left(a_{20}+1+a_{02}\right)^{2}} \\
& +\frac{4\left(a_{02}\right)^{2}+4 a_{02}+1}{\left(a_{20}+1+a_{02}\right)^{2}}=0 \\
& \lambda^{2}=\frac{-2 a_{02} a_{20}-3\left(a_{02}\right)^{2}+\left(a_{20}\right)^{2}-4 a_{02}-1}{\left(a_{20}+1+a_{02}\right)^{2}} \\
& \lambda_{1,2}= \pm \sqrt{\frac{-2 a_{02} a_{20}-3\left(a_{02}\right)^{2}+\left(a_{20}\right)^{2}-4 a_{02}-1}{\left(a_{20}+1+a_{02}\right)^{2}}}
\end{aligned}
$$
and to get a limit cycles the Eigen values must be pure imaginary i.e $\lambda_{1,2}= \pm i \beta$

So we can rewrite $\lambda_{1,2}=\alpha \pm i \beta \mathrm{w}$ where $\lambda_{1,2}=0 \pm$ $\frac{1}{a_{20}+a_{02}+1} \sqrt{2 a_{02} a_{20}+3\left(a_{02}\right)^{2}-\left(a_{20}\right)^{2}+4 a_{02}+1} i$$$
\beta=\frac{1}{a_{20}+a_{02}+1} \sqrt{2 a_{02} a_{20}+3\left(a_{02}\right)^{2}-\left(a_{20}\right)^{2}+4 a_{02}+1}
$$

Suppose that $R=2 a_{02} a_{20}+3\left(a_{02}\right)^{2}-\left(a_{20}\right)^{2}+4 a_{02}+1$

$$
\begin{gathered}
\beta=\frac{1}{a_{20}+a_{02}+1} \sqrt{R} \quad \text { if } a_{20}+a_{02} \neq-1 \\
2 a_{02} a_{20}+3\left(a_{02}\right)^{2}-\left(a_{20}\right)^{2}+4 a_{02}+1 \neq 0
\end{gathered}
$$

if $a_{02} \neq-a_{20}-1, a_{02} \neq \frac{a_{20}-1}{3}$

Now to get imaginary part $i=\sqrt{-1}$ we have two choices:if $2 a_{02} a_{20}+3\left(a_{02}\right)^{2}-\left(a_{20}\right)^{2}+4 a_{02}>-1$ then $R>0$ If $2 a_{02} a_{20}+3\left(a_{02}\right)^{2}-\left(a_{20}\right)^{2}+4 a_{02}<-1$ then $R<0$

The linear system (9) has the polynomial inverse integrating factor

$$
\begin{aligned}
& V(x, y)=\left(\left(a_{02}-a_{20}\right)(x+y)-1\right)\left(( 2 a _ { 0 2 } - 1 ) \left(a_{20}+\right.\right. \\
& \left.a_{02}+1\right)\left(a_{02} x^{2}-\left(a_{02}-a_{20}-1\right) x y+a_{02} y^{2}\right)+ \\
& \left.2 a_{02}\left(\left(a_{20}+a_{02}-1\right)(x+y)+1\right)\right) \text { and the first integral is }
\end{aligned}
$$




$$
H(x, y)=\frac{\begin{array}{c}
-\ln \left(x y+a_{0,2} y^{2}+a_{0,2} x^{2}+2 a_{0,2} a_{2,0}^{2} x y-4 x y a_{0,2}-a_{2,0} a_{0,2} y^{2}-x y a_{2,0}^{2}+2 a_{0,2}^{2} a_{2,0} x^{2}\right. \\
+5 a_{0,2}^{2} x y+2 a_{0,2}^{2} a_{2,0} y^{2}-2 a_{0,2}^{3} x y-a_{2,0} a_{0,2} x^{2}+2 a_{0,2}^{3} x^{2}+2 a_{0,2}^{3} y^{2}-3 a_{0,2}^{2} x^{2}-3 a_{0,2}^{2} y^{2} \\
\left.+2 a_{0,2} a_{2,0} x+2 a_{0,2} a_{2,0} y+2 a_{0,2} x+2 a_{0,2}^{2} y-2 a_{0,2} x-2 a_{0,2} y+2 a_{0,2}\right)
\end{array}}{\left(2 a_{0,2}-1\right)\left(3 a_{0,2}-1-a_{2,0}\right)\left(a_{2,0}+a_{0,2}-1\right)}-
$$

$$
\begin{aligned}
& \frac{2 \ln \left(a_{0,2} x+a_{0,2} y-a_{2,0} x-a_{2,0} y-1\right)}{\left(6 a_{0,2}^{2}-5 a_{0,2}-2 a_{0,2} a_{2,0}+a_{2,0}+1\right)\left(a_{0,2}-a_{2,0}\right)} \\
& +\frac{\ln \left(\left(a_{0,2}-a_{2,0}\right) x+a_{0,2} y-a_{2,0} y-1\right)}{\left(6 a_{0,2}^{2}-5 a_{0,2}-2 a_{0,2} a_{2,0}+a_{2,0}+1\right)\left(a_{0,2}-a_{2,0}\right)} .
\end{aligned}
$$

\subsection{Some applications of system (9)}

3.2.1 Case 1: If we take $a_{02}=0.9, a_{20}=0.01$ then the system become

$$
\begin{aligned}
& \dot{x}=x+0.01 x^{2}+x y+0.9 y^{2} \\
& \dot{y}=-y-0.9 x^{2}-x y-0.01 y^{2},
\end{aligned}
$$

Then the fixed points are $(0,0)$ and $\left(\frac{-1}{1.91}, \frac{-1}{1.91}\right)$

And the Eigen values for the point $\left(\frac{-1}{1.91}, \frac{-1}{1.91}\right)$ are: $\lambda_{1,2}= \pm 1.389941428$, and the phase portrait of the system (11) is

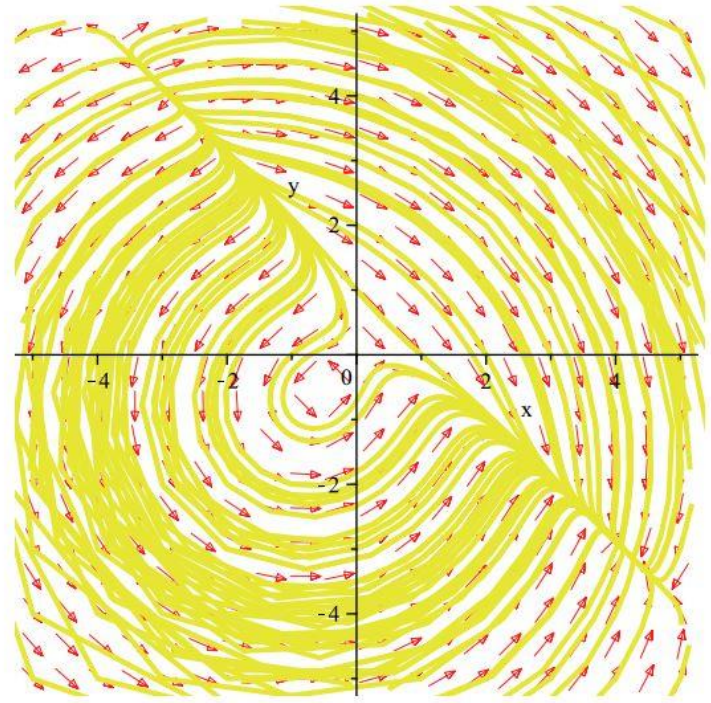

Figure 2. The phase portrait for the system (11)

3.2.2 Case 2: If we take $a_{02}=a_{20}=1$ then the system become

$$
\begin{aligned}
& \dot{x}=x+x^{2}+x y+y^{2} \\
& \dot{y}=-y-x^{2}-x y-y^{2},
\end{aligned}
$$

Then the fixed points are $(0,0)$ and $\left(\frac{-1}{3}, \frac{-1}{3}\right)$ and the Eigen values for the point $\left(\frac{-1}{3}, \frac{-1}{3}\right)$ are: $\lambda_{1,2}= \pm 1$ and the phase portrait of the system (12) is

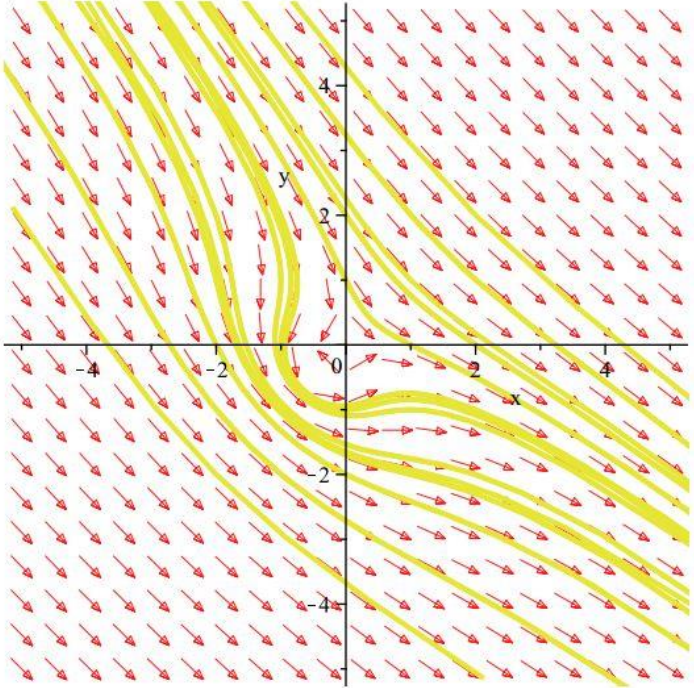

Figure 3. The phase portrait for the system (12)

The system (12) has the polynomial inverse integrating factor $V(x, y)=-x^{2}-x y-y^{2}-2 x-2 y-2$

and the first integral is

$$
\begin{gathered}
H(x, y)=y-\ln \left(x^{2}+x y+y^{2}+2 x+2 y+2\right)+x-x^{2} \\
-x y-y^{2}-2 x-2 y-2
\end{gathered}
$$

3.2.3 Case 3: If we take $a_{02}=1, a_{20}=0$ then the system become

$$
\begin{aligned}
& \dot{x}=x+x y+y^{2} \\
& \dot{y}=-y-x^{2}-x y
\end{aligned}
$$

Then the fixed points are $(0,0)$ and $\left(\frac{-1}{2}, \frac{-1}{2}\right)$ and the Eigen values for the point $\left(\frac{-1}{2}, \frac{-1}{2}\right)$ are: $\lambda_{1,2}= \pm 1.414$ and the phase portrait of the system (13) is

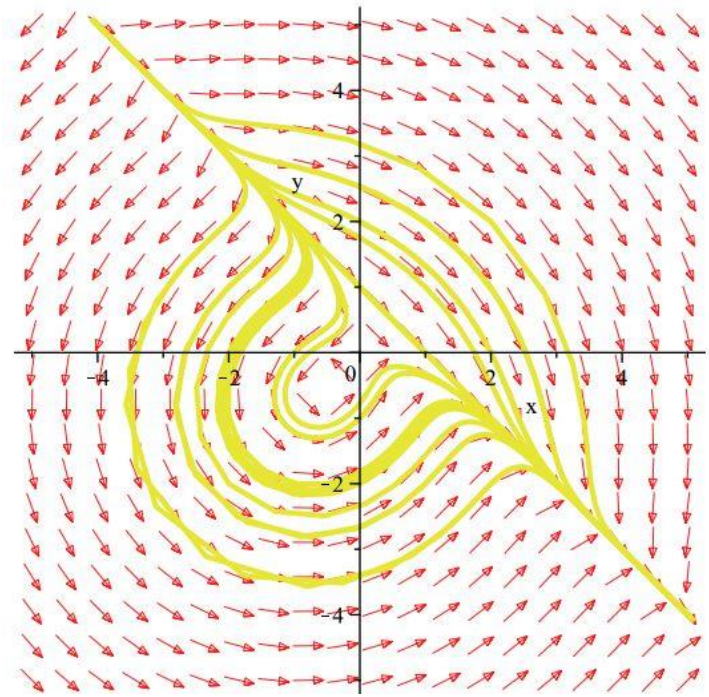

Figure 4 . The phase portrait for the system (13)

The system (13) has the polynomial inverse integrating factor $V(x, y)=2 x+2 y-2$ and the first integral is $H(x, y)=\frac{1}{4} y^{2}-\frac{1}{2} y-\frac{1}{2} \ln (x+y-1)-\frac{1}{4} x^{2}-\frac{1}{2} x$ 
3.2.4 Case 4: If we take $a_{02}=-1, a_{20}=1$ then the system becomes

$$
\begin{aligned}
& \dot{x}=x+x^{2}+x y-y^{2} \\
& \dot{y}=-y+x^{2}-x y-y^{2},
\end{aligned}
$$

Then the fixed points are $(0,0)$ and $(-1,-1)$ and the Eigen values for the point $(-1,-1)$ are: $\lambda_{1,2}= \pm \sqrt{3} i$ and the phase portrait of the system (14) is

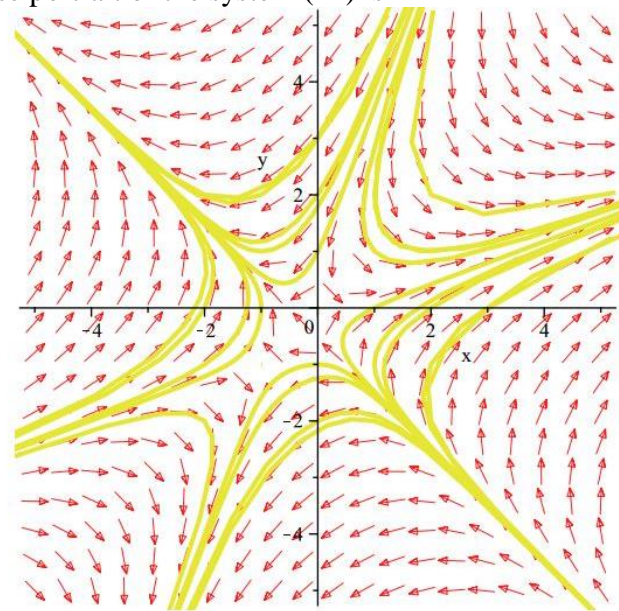

Figure 5. The phase portrait for the system (14)

The system (14) has the polynomial inverse integrating factor

$$
\begin{gathered}
V(x, y)=(-2 x-2 y-1)\left(-3 x^{2}+9 x y-3 y^{2}+2 x\right. \\
+2 y-2)
\end{gathered}
$$

and the first integral is

$$
\begin{gathered}
H(x, y)=\frac{1}{30} \ln (2 x+2 y+1)+\frac{1}{15} \ln \left(3 x^{2}-9 x y+3 y^{2}\right. \\
-2 x-2 y+2)
\end{gathered}
$$

3.2.5 Case 5: If we take $a_{02}=1, a_{20}=-1$ then the system become

$$
\begin{aligned}
& \dot{x}=x-x^{2}+x y+y^{2} \\
& \dot{y}=-y-x^{2}-x y+y^{2},
\end{aligned}
$$

Then the fixed points are $(0,0)$ and $(-1,-1)$ and the Eigen values for the point $(-1,-1)$ are: $\lambda_{1,2}= \pm \sqrt{5}$ and the phase portrait of the system (15) is

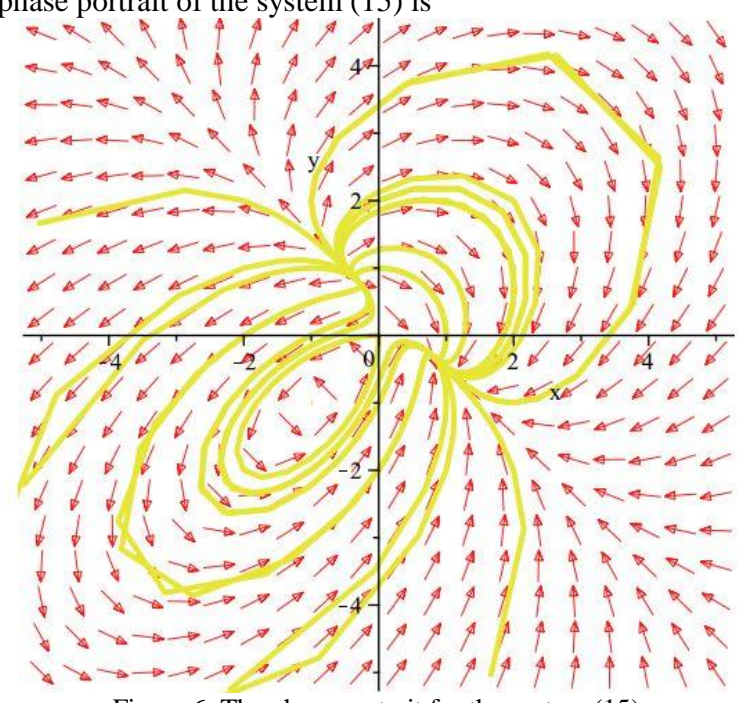

Figure 6. The phase portrait for the system (15)
The system (15) has the polynomial inverse integrating factor

$V(x, y)=(2 x+2 y-1)\left(-x^{2}+x y-y^{2}-2 x-2 y+2\right)$ and the first integral is

$H(x, y)=\frac{1}{3} \ln \left(x^{2}-x y+y^{2}+2 x+2 y-2\right)-\frac{1}{6} \ln (2 x+$

$2 y-1)$

Proof: To prove this Theorem 3.2 and all cases for finding polynomial inverse integrating factor is similar to the proof of Theorem 2.2.and Theorem 2.3.

\section{CONCLUSION}

We can conclude the following theorem.

From Theorem 2.2.and Theorem 2.3.

1) For system (1), if $P$ and $Q$ are homogenous polynomials of the same degree, then the polynomial function $V(x, y)=x Q-y P$ is a polynomial inverse integrating factor.

2) If a non-zero homogenous polynomial is an inverse integrating factor of the system (1), then it has no limit cycles.

3) All above cases for the system (9) has no limit cycles by Theorem 1.4 and Theorem 1.6.

\section{REFERENCES}

ArrowSmith, D.K. and Place, C.M. (1982). Ordinary differential equations. Mathematics series.

Calanchi, M. and Ruf, B. (2002). On the number of closed solutions polynomial ODE and a special case of Hilberts. Adv.Diff.Eq, $1978-2016$.

Chavarriga, J. (2004.). On the algebraic limit cycles of quadratic systems,. University Autonoma de Barcelona,

Chavarriga, J., Giacomini, H. and Gine, J. (1997). The null divergence factor. Publicacions Matematiques, 41-56.

Chavarriga, J., Giacomini, H. and Gine, J. (1999). On a new of bifurcation of limit cycles for a planar cubic system. Nonlinear Analysis, $139-149$

Chavarriga, J., Giacomini, H. and Gine, J. (2000). Polynomial inverse integrating factors. Ann.of Diff.Eqs, 320-329.

Chavarriga, J., Llibre , J. and Sorolla , J. (2001). Algebraic limit cycles for quadratic systems. preprint Universitat de Lleida.

Ferragut, A. (2006). Polynomial inverse integrating factors of quadratic differential systems and other results. Ph.D. thesis, University Autonoma de Barcelona.

Mustafa, Y. T., and Easif F. H. (2005). On limit cycles of a Certain Quintic System, Journal of Duhok University, Vol. 9, No. 1. 
بوونى زوّر رادهدارانى هه لكهراوهى هاوكوّلكهى تهواوكارى وتهواوكارييا ئيّى و نه بوونى ئامانجه خولهيهكان بوسيسته يله دوويى له رووتهختى يوتانه كاندا.

كورتيا ليّكولينى:

ئامانجا سهرهكى دثى قه كولينيّدا ثهكوّلينه دهربارهى بوونى زوّر رادهدارانى هه لكهراوهى هاوكوّلكهى تهواوكارى وتهواوكارييا ئيكى ، ونهبوونى ئامانجه

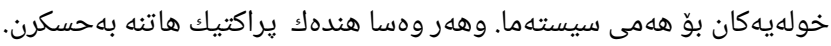
وجودية التكامل الاولى ومعكوس عامل التكامل المتعدد وعدم وجودية الدارات الغائية في الانظمة الحدودية من الدرجة الثانية.

خلاصة البحث: الهدف الرئيسى لهذا البحث هو دراسة وجودية معكوس عامل التكامل المتعدد و التكامل الاولى ، و دراسة عدم وجودية الدارات الغائية لكل الانظمة. بالاضافة ، تم اعطاء بعض التطبيقات. 